\title{
La Caisse des médecins fête ses 50 ans
}

\section{Ernst Gähler ${ }^{a}$ \\ Roger Scherrer ${ }^{b}$}

a Vice-président de la FMH, responsable du département Tarifs et conventions pour la médecine ambulatoire en Suisse

b FMH, chef de la division Tarifs et conventions pour la médecine ambulatoire en Suisse
Correspondance:

FMH / division Tarifs et

conventions pour la médecine ambulatoire en Suisse

Frohburgstrasse 15

CH-4600 Olten

Tél. 0313591230

Fax 0313591238

tarife.ambulant[at]fmh.ch

\section{Un partenaire fiable et digne de confiance}

La Caisse des médecins fête cette année ses 50 ans d'existence. La FMH profite de cette occasion pour lui adresser tous ses vœux et pour la remercier de sa précieuse collaboration. Fondée en 1964 par une poignée de médecins installés qui désiraient disposer de leur propre service de comptabilité, la Caisse des médecins reste aujourd'hui encore - malgré l'évolution des outils bureautiques et les progrès de la médecine - fidèle à son objectif premier: décharger les cabinets médicaux de leurs tâches administratives afin que les médecins puissent se concentrer sur la prise en charge de leurs patients. Pour cela, elle a développé toute une série de logiciels et de prestations permettant aux médecins de réduire les coûts et le temps investis dans les tâches administratives.

En tant que coopérative, la Caisse des médecins ne défend pas ses intérêts commerciaux propres, mais uniquement ceux de ses sociétaires, autrement dit les médecins suisses. Et comme ceux-ci sont à la fois clients et propriétaires de la Caisse des médecins, ils ont aussi la possibilité de participer au résultat. L'affiliation à la Caisse des médecins est ouverte à toutes les personnes habilitées à exercer en Suisse comme médecin indépendant.

La philosophie de la Caisse des médecins bénéficie à l'ensemble des médecins de même qu'à la FMH en sa qualité de fédération ainsi qu'aux sociétés cantonales de médecine et aux sociétés de discipline médicale. Fidèle à son principe «par les médecins pour les médecins», la Caisse des médecins soutient les activités en faveur de la qualité et s'engage tout particulièrement dans le domaine des tarifs ambulatoires notamment à travers le TARMED et la liste des analyses (principalement pour le tarif Point-of-Care)

Le département «Tarifs et conventions pour la médecine ambulatoire en Suisse» de la FMH a la chance de pouvoir compter sur les vastes connaissances du directeur exécutif Anton Prantl dans le domaine des tarifs mais aussi de l'informatique. Sur le plan financier également, notre département peut s'appuyer sur le soutien de la Caisse des médecins pour ses travaux. Cette dernière a œuvré au premier plan lors de l'introduction de la structure tarifaire TARMED de même qu'à la création de la société NewIndex SA. Au fil des ans, cette institution novatrice mise sur pied par la FMH et par les sociétés cantonales de médecine a su s'imposer, notamment lors de la phase de neutralité des coûts au moment de l'introduction du TARMED, durant laquelle elle s'est avérée être un soutien précieux et un fondement essentiel lors des séances mensuelles cantonales et régionales sur l'application des exigences en matière de neutralité des coûts. Aujourd'hui encore, il est indispensable que le corps médical dispose de ses propres données dans le cadre de la révision globale de la structure tarifaire TARMED, non seulement lors des négociations tarifaires mais aussi pour la validation des données et des bases de calcul des assureurs. Pour cela, Anton Prantl de la Caisse des médecins est aussi le vice-président du Conseil d'administration de NewIndex. Faisant preuve de clairvoyance, la Caisse des médecins a également participé aux côtés du corps médical à l'étude permanente des coûts (RoKo), dont les données forment encore aujourd'hui la base des modèles de coûts de la structure tarifaire TARMED.

Aujourd'hui plus que jamais, la FMH peut compter sur le précieux savoir-faire et le potentiel technique de la Caisse des médecins comme elle a pu le faire au cours de ces dix à quinze dernières années (notamment pour la révision de la liste des analyses, le modèle de rémunération sans marge de la propharmacie [nDMA], la révision globale du TARMED, etc.).

\section{Liste des analyses / laboratoire au cabinet médical}

Lors de la révision de la liste des analyses en 2009, nous avons pu participer aux discussions de manière particulièrement proactive grâce aux données et aux connaissances de la Caisse des médecins. A l'époque, nous n'avons malheureusement pas été entendus, et les conséquences n'ont pas tardé à se faire ressentir pour les laboratoires au cabinet. Malgré cela, le soutien de la Caisse des médecins nous a été très utile lors du monitorage de l'OFSP en vue du contrôle des résultats. C'est d'ailleurs sur la base des résultats de ce monitorage et du plan directeur «médecine de famille» que le conseiller fédéral Alain Berset a choisi d'opter pour le modèle de coûts de la FMH comme base économique pour le tarif Point-of-Care dans le domaine du laboratoire au cabinet. Développé par Anton Prantl et Roman Fried (directeur de l'Association pour le contrôle de qualité médical), ce modèle doit bientôt entrer en vigueur.

\section{Remise de médicaments sans marge}

Le reproche selon lequel la remise de médicaments par les médecins suscite des «incitatifs erronés» fait l'objet de discussions depuis des «décennies». Dans 
ce contexte, le corps médical a discuté de plusieurs options qu'il a tenté de mettre en ouvre. Avec le développement d'un système de remise exempte de marge, la Caisse des médecins a énormément contribué à l'apaisement de la situation et à la poursuite des négociations avec les assureurs. Malheureusement, animée par un désir effréné d'économies, santésuisse a fait échouer les négociations peu avant leur terme, alors que la solution interactive d'Anton Prantl aurait permis un gain d'efficacité important. Désormais, les discussions ont lieu avec un autre groupe d'assureurs sachant que ce modèle a non seulement convaincu l'OFSP mais également des parlementaires opposés à la propharmacie.

\section{Révision globale de la structure tarifaire TARMED}

Ici aussi, la FMH peut compter sur les connaissances approfondies et sur les compétences d'Anton Prantl, qui connaît parfaitement le TARMED et avait déjà développé les modèles de coûts avant son introduction en 2004. Désormais, cet ingénieur mécanicien de formation met ses connaissances à disposition de la FMH en tant qu'expert externe dans le cadre de la révision globale du TARMED en transmettant tout son savoir-faire aux collaborateurs de la division «Tarifs et conventions pour la médecine ambulatoire en Suisse». En collaboration avec la Caisse des médecins, nous avons pu développer notre propre navigateur tarifaire ainsi que différents instruments de simulation et disposons à présent de notre propre solution informatique qui ouvre de nombreuses perspectives d'avenir. Notre division est ainsi en mesure de tester et de montrer toutes les éventualités, de planifier l'avenir du tarif mais aussi de tester et de vérifier en toute confiance les idées des assureurs. Grâce à la Caisse des médecins et à son soutien, nous sommes en excellente voie. A l'occasion de ses 50 ans, la Caisse des médecins - fidèle à sa vision - a financé deux postes à plein temps en faveur du département «Tarifs et conventions pour la médecine ambulatoire en Suisse» pour les années 2014 et 2015 afin que celui-ci dispose des ressources nécessaires pour finaliser la révision de la structure tarifaire d'ici fin 2015.

\section{Les plus vifs remerciements de la FMH à la Caisse des médecins}

La collaboration entre la FMH et la Caisse des médecins bénéficie aussi bien à l'un qu'à l'autre acteur. Les médecins en tant que sociétaires de la Caisse des médecins, la FMH et son département «Tarifs et conventions pour la médecine ambulatoire en Suisse» ont la chance de pouvoir récolter les fruits de cette collaboration constructive née voilà plusieurs décennies. Les jalons sont désormais posés. La FMH et la Caisse des médecins sont tous deux propriétaires à part entière des modèles de coûts, de leur propre navigateur tarifaire, et des modèles de simulation. Nous tenons donc à remercier de tout cœur la Caisse des médecins et nous lui souhaitons de poursuivre sur cette voie en nous réjouissant d'ores et déjà de continuer à collaborer avec elle dans l'intérêt des médecins.

\section{Un grand merci et nos meilleurs vœux!}

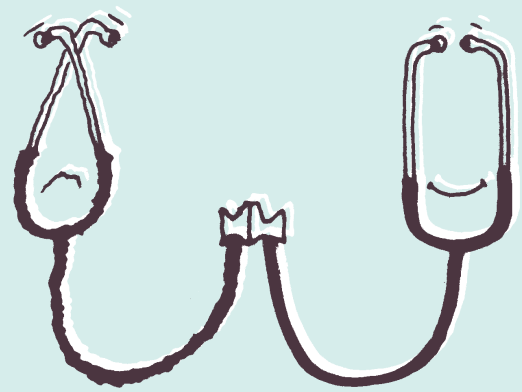

Aimin

\section{Et soudain, vous aussi! Les crises touchent aussi les médecins.}

Acceptez de l'aide. Contactez ReMed.

Assistance téléphonique $24 \mathrm{~h}$ sur 24

0800073633 help@swiss-remed.ch

08000 ReMed www.swiss-remed.ch 